\title{
An efficient neuro-fuzzy based segmentation of normal tissues in brain MRI (BMRI) using extensive feature set
}

\author{
M.Y.Bhanumurthy ${ }^{1}$ and Koteswararao Anne ${ }^{2}$ \\ ${ }^{1}$ Dept.of ECE, Vasireddy Venkatadri Institute of Technology, Guntur-522006, A.P, INDIA; \\ ${ }^{2}$ Dean Academics, V.R.Siddhartha Engineering College, Vijayawada, A.P, INDIA; \\ mybhanu@gmail.com; raoanne@gmail.com
}

\begin{abstract}
Brain tissue Segmentation from the MRI images is having significance in the medical research field. The accurate Segmentation of the normal as well as the abnormal tissues is the complex assignment in this process. In this paper, a technique named Neuro-Fuzzy Based Segmentation (NFBS) is proposed for segmenting the normal features such as White Matter (WM), Gray Matter (GM) and Cerebro-Spinal Fluid (CSF) in the MRI Brain images. (1) Feature extraction (2) Classification (3) Segmentation are the three stages offered in this work. At first, the features such as energy, entropy, homogeneity, contrast and correlation from MRI Brain Images are extracted. Next, by utilizing Neuro-Fuzzy classifier, the Classification process is carried out and for this process, the feature set is specified as the input. From the outcome of Classification, the images are categorized into normal as well as abnormal. The further procedure Segmentation is performed according to this outcome only. The normal MRI images are segmented into normal tissues like White Matter (WM), Gray Matter (GM) and Cerebro-Spinal Fluid (CSF). All the tissues are individually segmented by special methods such as Gradient method, Orthogonal Polynomial Transform method. Utilizing MATLAB platform, the implementation of the proposed technique is made. The experimentation is carried out on the MRI Brain Images by BrainWeb data sets. The performance of the proposed technique is assessed with the help of the metrics namely FPR, FNR, Specificity, Sensitivity and Accuracy. Therefore, using our proposed techniques with enhanced classification, the normal tissues of MRI Brain images are segmented accurately.
\end{abstract}

Keywords:- Segmentation, Classification, Neuro-Fuzzy Logic, Normal and abnormal tissues, Gradient Method, Orthogonal Polynomial Transform.

\section{Introduction}

The brain is the frontal most part of the central nervous system. It forms the Central Nervous System (CNS) along with the spinal cord. The Cranium, a bony box in the skull guards it. Because of our brain in practical we do lots of things like, to think, act, reason, walk, talk, the list is never-ending. Brain Tumors are one of the syndrome caused in the brain. In an uncontrolled behavior cells reproduces themselves that causes abnormal growth which is called tumor. A benign brain tumor contains benign (harmless) cells and has different manner. This tumor can be cured only by surgery. A malignant brain tumor is very critical. Because of its location and it contains of cancer cells it may be termed as malignant. A malignant 
brain tumor invented of cancerous cells which possibly will spread or begin in other locations in the brain or spinal cord. Hence it cannot function correctly as it can attack and destroy healthy tissues.

By means of Magnetic Resonance Imaging (MRI) doctors and researchers can examine noninvasively the structure and function of the brain. In fact, the MRI image is a thin horizontal slice of the brain. The white area at lower left is the tumor. It looks white as MRI scans develops the tissue variation. In reality, the tumor is on the right side of the brain. In recent times, various people utilize the MRI data to explore the relation among white matter development and neural diseases particularly the anatomy image is combined with those images from diffusion tensor imaging. And the accuracy of segmenting white matter is a key problem when it is utilized to lead the fibre tack [1-2]. Attention deficit hyperactivity disorder (ADHD) [3] is as well required to segment white matter.

Various algorithms have been proposed for brain MRI segmentation recently. Different algorithms for segmenting MRI of data [4-8] are watershed algorithm, eSneke algorithm and genetic algorithm. Those algorithms are based on the homogeneity of image. Actually, we have to solve the problem with new technique as the intensity in homogeneity is impact on every image. The majority well-liked techniques are consisted thresholding [8], region-growing [9] and clustering. The complete mechanical intensitybased algorithms include high sensitivity to different noise artifacts that is intra-tissue noise and intertissue intensity contrast reduction. Thresholding is very ease and competence. The intensity histogram of the image is bimodal if the target is plainly noticeable from background, and by simply selecting the valley bottom as the threshold point, it can be easier to obtain the optimal threshold. On the other hand, in the majority of real images, there are not plainly noticeable marks among the target and the background. Clustering is the majority well-liked approach for segmentation of brain MR images and naturally executes better than the other techniques [10]. Wells [9] buildups a latest statistical approach based on the expectation-maximization (EM) algorithm, however the results are too reliant on the initial values, very time consuming and just looking for local maximum point.

Segmentation is an significant implement in medical image processing and it has been helpful in several applications, namely: detection of tumors, detection of the coronary border in angiograms, surgical planning, measuring tumor volume and its response to therapy, automated classification of blood cells, detection of micro calcifications on mammograms, heart image extraction from cardiac cine angiograms, etc [11]-[14]. It may be helpful to categorize image pixels into anatomical regions in some applications such as bones, muscles, and blood vessels, while in others into pathological regions, such as cancer, tissue deformities, and multiple sclerosis lesions. To detach an image into regions that are homogeneous with respect to one or more characteristics is the primary objective in segmentation process [10]. The aim is to separate the whole image exactly into sub regions included gray matter (GM), white matter (WM) and cerebrospinal fluid (CSF) spaces of the brain [15] in magnetic resonance (MR) images processing. As, in a number of neurological disorders like multiple sclerosis (MS) and Alzheimer's disease, the volume changes in total brain, WM, and GM can give major notification about neuronal and axonal loss [16].

The remaining of the paper is prepared as follows: After this Introduction part the next section surveys several works that have already segmenting the tissues in the MRI Brain images. Section 3 explains our proposed technique of segmenting the tissues of BMRI images utilizing Neuro-Fuzzy Classifier. The 
M.Y.Bhanumurthy and Koteswararao Anne; An efficient neuro-fuzzy based segmentation of normal tissues in brain MRI (BMRI) using extensive feature set, Journal of Biomedical Engineering and Medical Imaging, Volume 1, No 5, Oct (2014) , pp 1-22

outcomes regarding the performance of our proposed work are specified in the Section 4 and as a final point; our paper is summed up with the conclusion part in Section 5.

\section{Literature Survey}

Researchers proposed for many researches for the brain image segmentation. A short access of several researches is offered here. Arnaldo Mayer and Hayit Greenspan [20] have offered an automated segmentation framework for brain MRI volumes based on adaptive mean-shift grouping in the joint spatial and intensity feature space. The technique was authorized both on simulated and real brain datasets, and the outcomes were compared with state-of-the-art algorithms. The benefits over intensity based GMM EM schemes as well as additional state-of-the-art techniques were established. Moreover they proved that by means of the AMS framework, segmentation of the normal tissues is not degraded by the presence of abnormal tissues. The algorithm gave good outcomes on noisy and biased data while only a rudimental bias field improvement part executed and no spatial prior was extracted from an atlas. And thanks to the adaptive mean-shift ability to work with non-convex clusters in the joint spatial intensity feature space and also the mean-shift noise smoothing behavior.

Mert R. Sabuncu et al. [21] have examined a generative model that guides to label fusion style image segmentation techniques. They originated several algorithms that merge transmitted training labels into a single segmentation estimate in the proposed framework. An expert gave a dataset of 39 brain MRI scans and equivalent label maps and we analytically compared these segmentation algorithms with Free Surfer's broadly-used atlas-based segmentation tool. Their outcomes established that the proposed framework yields an accurate and robust segmentation tools that are employed on large multi-subject datasets. They utilized one of the enhanced segmentation algorithms to calculate hippocampal volumes in MRI scans of 282 subjects, in a second experiment. A assessment of these measurements across clinical and age groups signifies that the proposed algorithms were adequately sensitive to detect hippocampal volume variations related with earlier Alzheimer's disease and aging.

By utilizing a subject-specific tissue probabilistic atlas produced from longitudinal data, Feng Shi et al. [22] have offered a framework for presenting neonatal brain tissue segmentation. Proposed method has received the benefit of longitudinal imaging study in their system, i.e., by means of the segmentation outcomes of the images obtained at a late time to direct the segmentation of the images obtained at neonatal stage. Compared to the two population-based atlases the testing outcomes revealed that the subject-specific atlas has better performance. And moreover the proposed algorithm attained comparable performance as manual raters in neonate brain image segmentation. By attaining optimal segmentation results in a broad range of $0.3-0.6$, the atlas sharpness parameter has been shown robust appearance. For the selection of late time-point image, the segmentation accuracy remains alike when the atlas was developed by either one-year-old or two-year-old image.

Juin-Der Lee et al. [23] have offered the most statistical segmentation methods in the literature and have presumed that either the intensity allocation of every tissue variety was Gaussian, or the logarithmic transformation of the raw intensity was Gaussian. As an alternative of setting up further classes to model "mixels," they proposed a power transformation approach to carry out automatic segmentation of brain MR images into CSF, GM, and WM. By instinct it was understandable that the familiar Box-Cox power transformation model was capable to give a statistically significant and helpful solution to proposed difficulty. To include both Gaussian intensity distributions as well as non-Gaussian 
distributions, the shape parameter utilized to widen the traditional Gaussian mixture models. And the parameters can be expected by means of the EM algorithm. They authorized the approach against four real and simulated datasets of normal brains from the IBSR and BrainWeb. Testing's on real data from the IBSR have shown that compared with other techniques utilized presently, the proposed approach attains higher Jaccard indexes. The power transformation approach maintains the simplicity of the Gaussian mixtures, and in addition it has the prospective to simplify the multivariate versions personalized for segmentation by means of multi-modality images.

Dalila Cherifi et al. [24] have illustrated normal tissue's recognition than tumor extraction (applied for GBM and MS diseases). To detach the abnormal tissues they have offered brain recognition techniques. Based on thresholding utilized for tumor extraction (GBM and MS diseases) they have proposed and applied the technique. They have originated that the local thresholding provides a good outcomes comparing with the others. They have accomplished that when they merge median filter, local thresholding and post processing in such a way that the resultant algorithm is tougher. For tissue recognition and tumor extraction they have executed categorization based on EM segmentation technique. Comparing with thresholding particularly for detecting the small regions of necrotizing tissue which was inside Anaplastic cells (pseudo-Palisading necrosis) for GBM tissue, proposed technique provided us better outcomes; and it mainly for the reason that of parameters that utilized in this algorithm.

Nagesh Vadaparthi et al. [25] have offered a paper in which particular cases like Acoustic neuroma, it was presumed that there was an option of hearing loss, dizziness and other symptoms associated to brain. Surgery can cure various acoustic neuromas. Hence, it was required to segment the image more correctly, which assisted to recognize the damaged tissues to be repaired and can be corrected by surgery. And so a new novel segmentation algorithm based on Skew Gaussian distribution was proposed in proposed paper, which assisted to recognize the tissues more correctly. Because of the basic structure of Skew Gaussian distribution it was suitable for symmetric and asymmetric distribution. The performance evaluation was succeeded by utilizing quality metrics. The outcomes proved that, proposed developed algorithm outperforms the existing algorithm. Various models were exploited to recognize the diseases, although due to the utility of non-ionizing radiation, MRI brain segmentation has achieved popularity over the other models.

Usually noise is generated by equipments, environment and also the performance of operator in MRI Brain images which creates serious incorrectness in the outcome of Segmentation procedure. Several of the unverified techniques did not deal with the intensity and in-homogeneity artifacts. And also the managed techniques undergo with the shortcomings of manual intervention for providing a priori notification. Pathological tissues demonstrate inconsistency in their structures. The shape of these tissues is deformable, the location of them across the patients may differ extensively, and also their characteristics of texture and intensity might vary. These difficulties of the existing schemes are generally un-solvable. A few techniques do not consider the large deformation of brain structures. The practice of brain atlas might show the way to false learning, though such deformations occurs. 


\section{Proposed Methodology}

Initially, the input BMRI images are given to our proposed work NFBS. The feature sets are extracted from these input images. From these feature sets, the images are classified into two kinds of tissues normal and abnormal using the Neuro-Fuzzy classifier. Then the normal tissues are segmented using various techniques. The normal tissues that are segmented are White Matter (WM), Gray Matter (GM) and Cerebro-Spinal Fluid (CSF). The proposed work is illustrated in Fig. 1.

\subsection{Phases of NFBS}

For our proposed method, to segment the BMRI images effectively, the three phases are presented which are as follows:
I. Feature set Extraction
II. Neuro-Fuzzy classifier based Classification
III. Classified tissue's Segmentation

\subsubsection{Phase I: Extraction of extensive feature sets}

In order to classify the given Brain MRI images, the features from these MRI images are initially extracted. In our work, the statistical features such as Energy, Entropy, Homogeneity, Contrast and Correlation are extracted from these input BMRI images.

\section{Energy}

Energy is also called as uniformity. Within $[0,1]$ the range of energy is presented. The value of energy for a constant image is 1 . The equation for finding energy is,

$$
E_{g}=\sum_{i, j} p(i, j)^{2}
$$

where, $p(i, j)$ is the pixel value at the point $i, j$ of the BMRI image of size $M \times N$. 


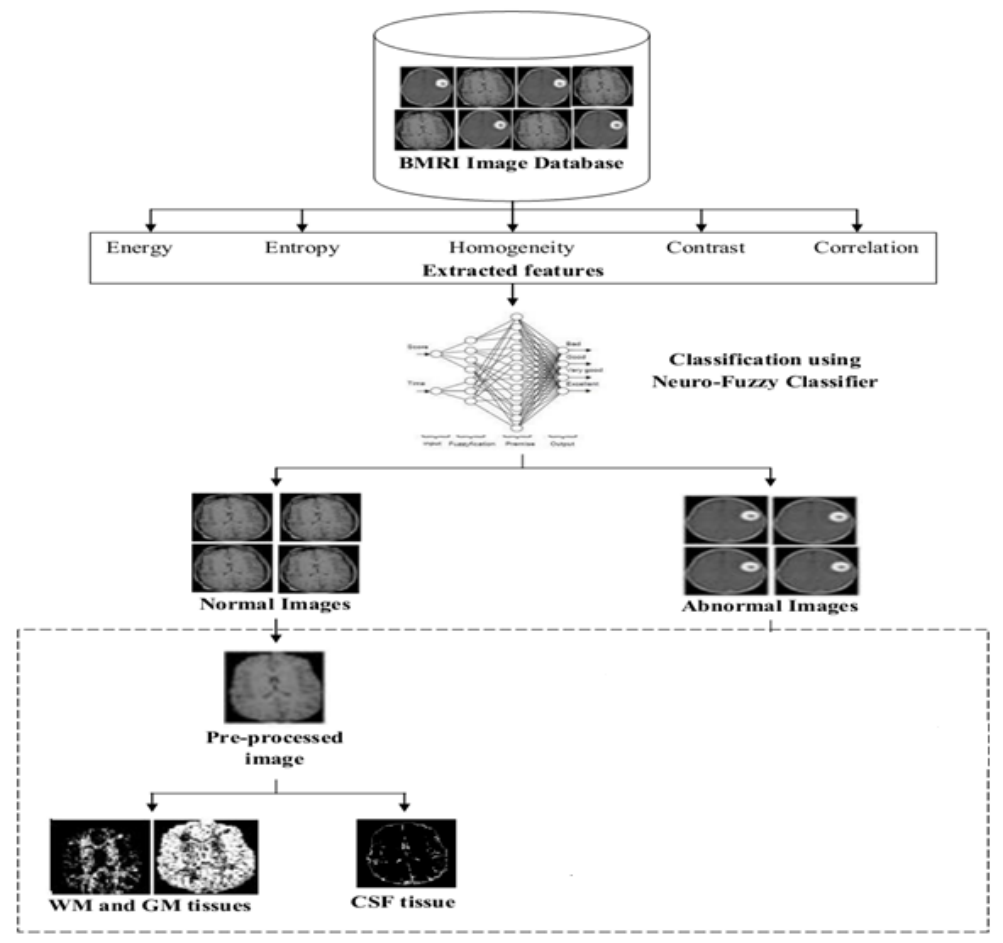

Figure 1: Proposed NFBS block diagram

\section{Entropy}

Entropy helps to characterize the texture of the BMRI image and to find out the distribution variation in a region of the image. Entropy is calculated as follows,

$$
E_{p}=\sum_{k=0}^{G-1} P b_{k}\left(\log _{2} P b_{k}\right)
$$

where, $P b_{k}$ is the probability of kth gray level and the kth gray level is calculated using $\frac{Z_{k}}{M \times N}$. In this, $Z_{k}$ represents the total number of pixels in the image with kth gray levels. $G$ indicates the total number of gray levels.

\section{Homogeneity}

Homogeneity provides the closeness of the elements. It has the range of $[0,1]$. It is computed as,

$$
H_{m}=\sum_{i, j} \frac{p(i, j)}{1+|i-j|}
$$

\section{Contrast}

The intensity contrast between a pixel of an image and the neighbor of that pixel throughout the whole image is defined by this Contrast measure. For a constant image, the contrast is set as 0 . It is specified as, 


$$
C_{n}=\sum_{i, j}|i-j|^{2} p(i, j)
$$

\section{Correlation}

It tells about the correlation between a pixel and its neighbor over the whole BMRI image. Its range is [$1,1]$. If an image has the value of correlation as 1 means, then it indicates the perfectly positively correlated image and if it is -1 means, then it shows the image is perfectly negatively correlated. The correlation of a constant image is not a number.

$$
C_{r}=\sum_{i, j} \frac{\left(i-\mu_{i}\right)\left(j-\mu_{j}\right) p(i, j)}{\sigma_{i} \sigma_{j}}
$$

where, $\mu_{i}, \mu_{j}, \sigma_{i}, \sigma_{j}$ are the means and standard deviations of the partial probability density functions $P_{i}, P_{j}$.

$$
\begin{gathered}
\text { Mean, } \quad \mu=\frac{1}{M N} \sum_{i=1}^{M} \sum_{j=1}^{N} p(i, j)^{2} \\
\text { Standard Deviation, } \sigma=\sqrt{\frac{1}{M N} \sum_{i=1}^{M} \sum_{j=1}^{N}(p(i, j)-\mu)^{2}} \\
\text { Variance, } \quad \operatorname{Var}=\sqrt{\sigma}
\end{gathered}
$$

Thus the feature correlation of the images is calculated using the mean and variance equations. Hence, all the feature sets $E_{g}, E_{p}, H_{m}, C_{n}$, and $C_{r}$ are extracted from the input BMRI images directly.

\subsubsection{Phase II: Classification using Neuro-fuzzy classifier}

The BMRI images are classified using the Neuro-Fuzzy classifier. The extracted features $E_{g}, E_{p}, H_{m}, C_{n}$, and $C_{r}$ are given as the input to the Neuro-Fuzzy Classifier for classifying all the given BMRI images into 2 classes such as Normal BMRI images and Abnormal BMRI images. The Neurofuzzy system has a three-layered architectural design; the following diagram fig. 2 shows the basic structure of the neuro-fuzzy classifier system. Neuro-Fuzzy classifier is a fuzzy based system that is trained by a learning algorithm derived from Neural Networks. The learning algorithm only performs on the local information and provides the local modifications in the fuzzy system. In general, a neuro-fuzzy system generates very powerful solutions instead of using the system components individually. 


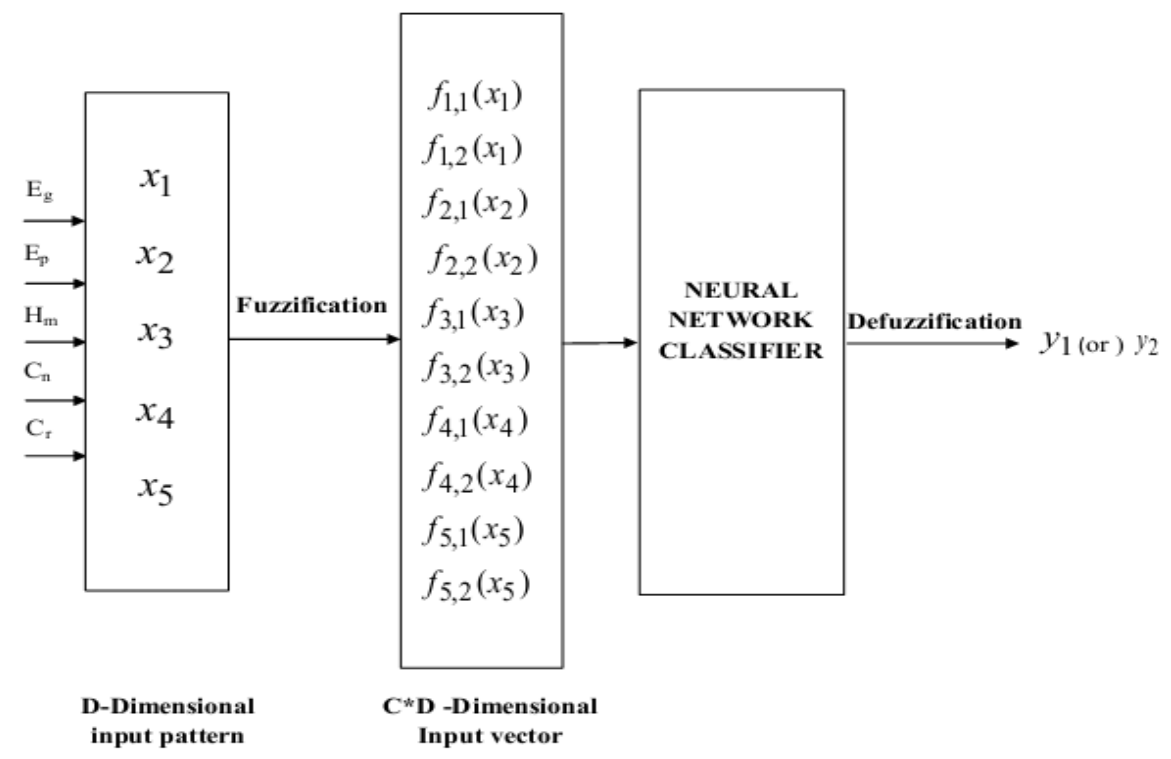

Figure 2: Architecture of Neuro-Fuzzy classifier

\section{Fuzzification}

The input values are the extracted features $E_{g}, E_{p}, H_{m}, C_{n}$, and $C_{r}$, which are received by the system as the input and then these input feature values are fuzzified using membership functions (MF) that facilitates the membership of each features to different classes. The hidden and inter-related information are extracted from the features to the classes through the MF, which leads to get more accuracy of the classification phase using Neuro-fuzzy classifier. The membership matrix comprises with 5 rows and 2 columns, in which the number of rows is equal to the number of features and the number of columns is equal to the number of classes.

The membership matrix $f_{d, c}\left(x_{d}\right)$ produced, describes the degree of belonging of different features ( $D$ ) to different classes $(C)$.

where, $x_{d}-d^{\text {th }}$ feature value of pattern $X$.

$$
\begin{aligned}
& d-1,2, \ldots, D \text {, here number of features is } 5 . \\
& c-1,2, \ldots, C \text {, here number of classes is } 2 .
\end{aligned}
$$

The representation of pattern is as follows,

$$
X=\left[x_{1}, x_{2}, x_{3}, x_{4}, x_{5}\right]^{T}
$$

In this a $\pi$-type MF is used as the membership function to classify the images. It is a bounded function having a shape similar to that of the Gaussian/exponential function. The $\pi$-type MF has fuzzifier ( $m$ ) as the parameter that can be tuned corresponding to the need of the problem. This controls the generalization capability by choosing a proper value of the fuzzifier $m$ and gives more flexibility for 
classifying the images. The steepness of the Gaussian function is controlled by varying the fuzzifier value, which is defined as follows,

$$
\pi(X ; a, r, b)=\left\{\begin{array}{lr}
0, & \text { if } X \leq a \\
2^{m-1}\left[\frac{(X-a)}{(r-a)}\right]^{m}, & \text { if } a<X \leq p \\
1-2^{m-1}\left[\frac{(r-X)}{(r-a)}\right]^{m}, & \text { if } p<X \leq r \\
2^{m-1}\left[\frac{(X-r)}{(b-r)}\right]^{m}, & \text { if } r<X \leq q \\
1-2^{m-1}\left[\frac{(b-X)}{(b-r)}\right]^{m}, & \text { if } q<X<b \\
0, & \text { if } X \geq b
\end{array}\right.
$$

The value $r$ is the center of MF, and $r=\frac{(p+q)}{2}$, in which $p$ and $q$ are the two crossover points. The membership function after the fuzzification process is expressed for a pattern $X$ as follows,

$$
F(X)=\left[\begin{array}{ll}
f_{1,1}\left(x_{1}\right) & f_{1,2}\left(x_{1}\right) \\
f_{2,1}\left(x_{2}\right) & f_{2,2}\left(x_{2}\right) \\
f_{3,1}\left(x_{3}\right) & f_{3,2}\left(x_{3}\right) \\
f_{4,1}\left(x_{4}\right) & f_{4,2}\left(x_{4}\right) \\
f_{5,1}\left(x_{5}\right) & f_{5,2}\left(x_{5}\right)
\end{array}\right]
$$

All rows and columns in the membership matrix are cascaded and converted into a vector by this cascading. This generated vector $V_{i}$ is given as the input to the Neural Network (NN).

\section{Neural Network}

In this, Feed Forward Multi-layer Perceptron classifier is used which has three layers such as input layer, hidden layer and output layer.

The total number of input nodes of the $\mathrm{NN}$ is equal to the product of the number of features and classes. In this paper the product of 5 features and 2 classes is 10 , which is the number of input nodes of the NN. The total number of output nodes from the NN is same as that of the number of classes, and here 2 output nodes are generated from the NN. The total number of hidden nodes is equal to the square root of the product, of the number of input nodes and output nodes. The structure for the Neural Network is given in fig.3. 


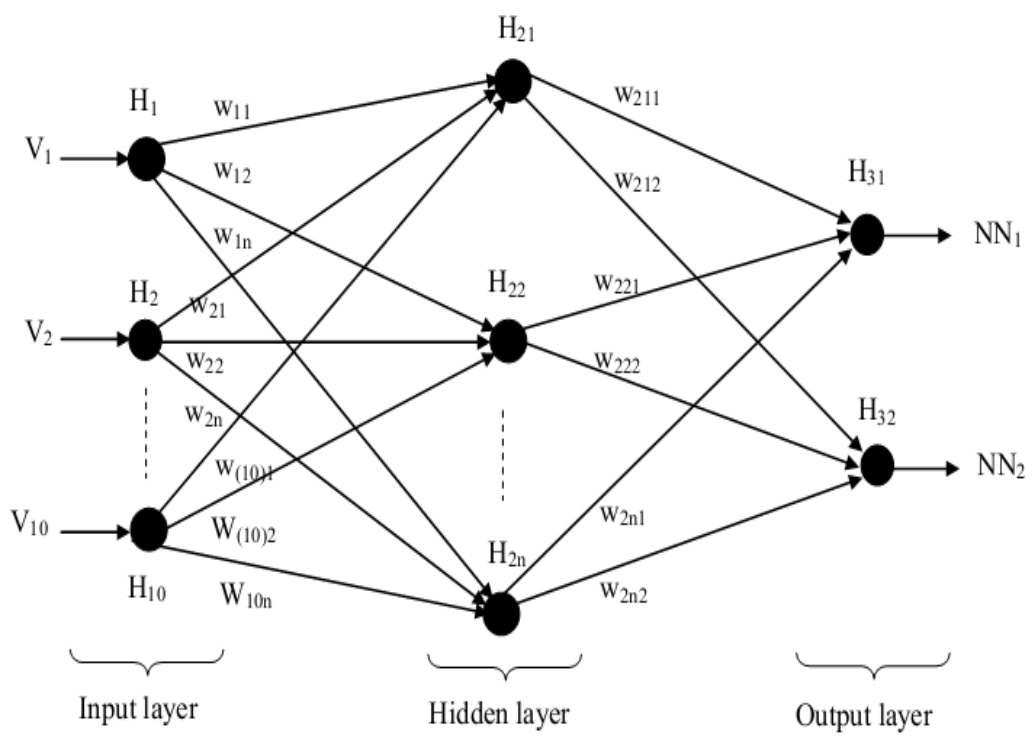

Figure 3: Neural Network classifier

\section{Defuzzification}

Then the defuzzification process is carried out on the output nodes of NN, by performing a MAX (maximum) operation. The output is a single value, $y_{1}$ or $y_{2}$ for a given BMRI image. From this value, we can able to classify whether the given input BMRI image is normal or abnormal.

\subsubsection{Phase-III: Segmentation of classified tissues}

Utilizing Neuro-fuzzy classifier the BMRI images are classified and after that the images are comprised only in any of the two different images normal and abnormal or pathological images. From the normal images, the normal tissues like White Matter (WM), Gray Matter (GM), and Cerebro-Spinal Fluid (CSF) are segmented. Prior to the segmenting of these normal images, one of the procedure named as preprocessing is carried out only on the normal images, not on the abnormal images. Almost it is very simple to place the CSF tissue of the normal BMRI images in the area that surrounds the cortex by use of the pre-processing technique. For segmenting the tissues, the segmentation stage is sustained after that on both the classified images efficiently.

\section{Pre-processing of normal BMRI images}

In order to apply the Morphological operations [26] on the images, firstly, the classified BMRI normal images are transformed into gray scale images. Next, the brain cortex is stripped in the gray scale image by means of Region Based Binary Mask Extraction procedure. In general, the brain cortex can be observed as the ring around the brain tissues in BMRI images. The Skull Stripping technique is used for these images to eradicate the ring that surrounds the brain tissues. Utilizing Skull Stripping technique, normal BMRI image is attained after the pre-processing is denoted as $I_{S S}$. 


\section{BMRI image tissue's Segmentation}

The segmentation of the tissues of both the normal and abnormal images takes place after the normal BMRI images gets pre-processed. In the normal BMRI images, the normal tissues namely WM, GM and CSF are used for segmentation. Herein, WM and GM are segmented by means of Gradient technique and CSF is segmented by use of Orthogonal Polynomial Transform (OPT) technique.

\section{Segmentation of WM and GM}

For segmenting the White Matters and Gray Matters, the pre-processed skull stripped image $I_{S S}$ is subjected into gradient technique. The Gaussian Convolution filter that utilized in this technique makes the image $I_{S S}$ into smoothed image $I_{S}$. After that, the smoothed image $I_{S}$ is subjected with Gradient operation. The gradient of two variables $x$ and $y$ are specified as follow:

$$
\nabla I_{S}(x, y)=\frac{\partial I_{S}}{\partial x} \hat{e}+\frac{\partial I_{S}}{\partial y} \hat{f}
$$

The gradient values are useful to mark the current edges in the image that are specified in the following equations (10) and (11).

$$
\begin{gathered}
S=x_{(e)}{ }^{2}+y_{(f)}{ }^{2} \\
E M=\frac{1}{(1+S)}
\end{gathered}
$$

The process of Binarization is then carried out on the edge marked image $E M$. In this Binarization procedure, the value of gray level of every pixel in $E M$ image is estimated by means of a global threshold value $T_{g}$. The resultant binarized image after the Binarization process is $I_{B}$.

By use of Morphological Opening and Closing operation, the small holes and small objects from the image $I_{B}$ is eliminated. Currently, in our work, the WM and GM normal tissues of normal BMRI images are segmented by means of the intensity values.

$$
I_{W G}= \begin{cases}W M, & \text { if } I_{B_{i}}=1 \\ G M, & \text { if } I_{B_{i}}=0\end{cases}
$$

The exacting part is segmented into White Matter, if the intensity value of the image part is one and then it is considered as Gray Matter part, if the intensity value is zero, and subsequently the images are segmented according to the equation (12).

\section{Segmentation of CSF}

CSF tissue from the image $I_{S S}$ is segmented by Orthogonal Polynomial Transform (OPT) using the formula given below: 


$$
I_{C S F}=\operatorname{Sin}\left(\frac{I_{S S_{(i)}}{ }^{3}}{100}\right)^{2}+\left(0.05 * \operatorname{rand}\left(\left|I_{S S}\right|\right)\right)
$$

At present, the CSF tissue $I_{C S F}$ from the normal image is segmented efficiently. Therefore, the normal tissues WM, GM and CSF are segmented from the pre-processed image $I_{S S}$.

\section{Results and Discussions}

Our proposed NFBS for the effective segmentation of normal tissues is implemented using the MATLAB platform on the Brain MRI images from the dataset. The data set description is given below in detail.

\subsection{Dataset Description:}

BrainWeb dataset is utilized with different BMRI images for our proposed work. Based on standard tissue segmentation mask, BrainWeb datasets give MRI brain images with unreliable image quality. The datasets are too based on an anatomical structure of a normal brain, which results from the tasks of registering and preprocessing of 27 scans from the same individual with segmentation. Different kinds of tissues are well identified in this dataset, both the types of tissue memberships "fuzzy" and "crisp" are assigned to each voxel. The sample Brain MRI images from the BrainWeb data set are specified in the fig. 4 given below.
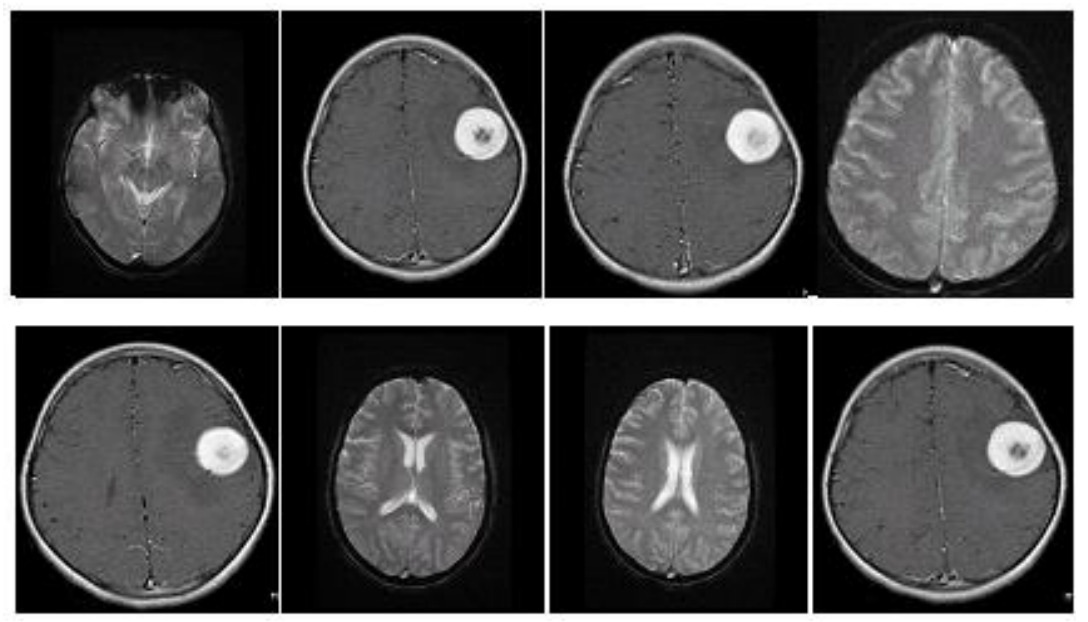

Figure 4: Sample BMRI images from dataset.

Our proposed work is estimated by means of 23 BrainWeb MRI images. 9 images are normal and the remaining 14 images are abnormal among $23 \mathrm{MRI}$ images. At first, the BMRI images from this dataset is taken and offered to the procedure of our proposed NFBIS. Five of the statistical characteristics from these BMRI images are extracted. Then for the categorization of images these extracted features are utilized. In order to categorize the particular images into normal and abnormal images, Neuro-Fuzzy classifier is utilized as the classifier in this proposed NFBIS work.

The classified normal and abnormal images are specified in the fig. 5 given below. 

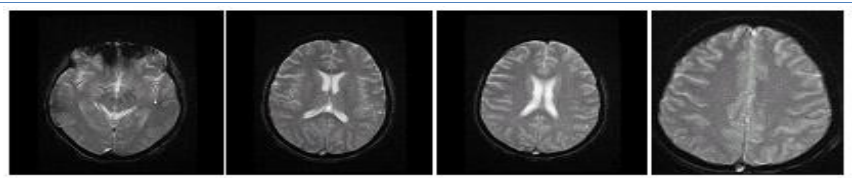

(a)

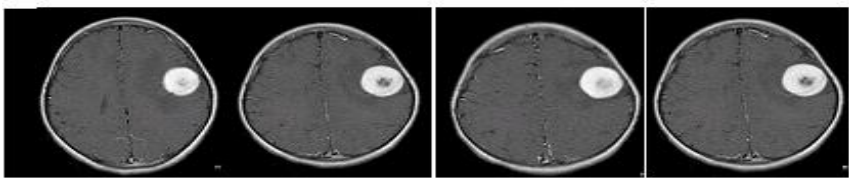

(b)

Figure 5: Normal and Abnormal Images

Subsequently, the Segmentation is performed on the classified BMRI images. The first step in the segmentation of normal images is the Skull Stripping Method which is carried out on the classified images by means of pre-processing. The normal image after pre-processing is specified in fig. 6 given below:
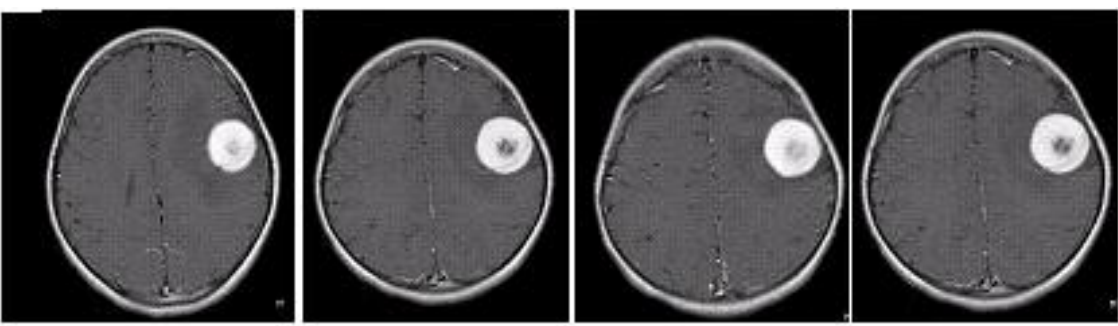

Fig. 6: Normal BMRI images after pre-processing
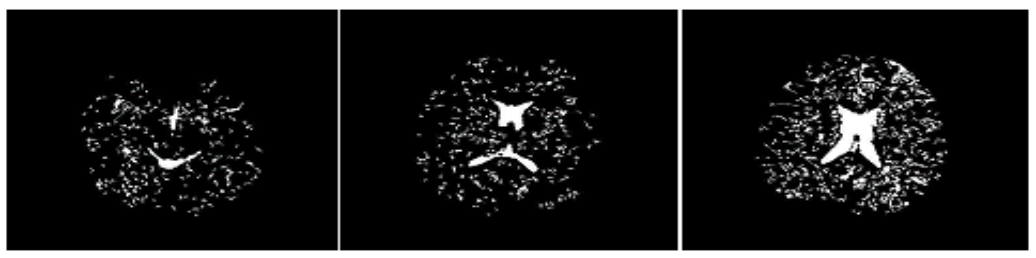

(a)
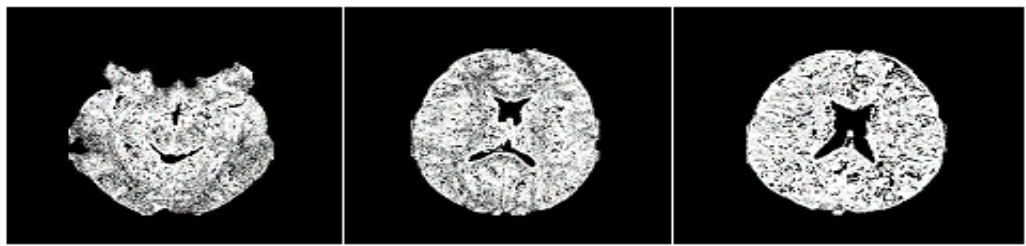

(b)
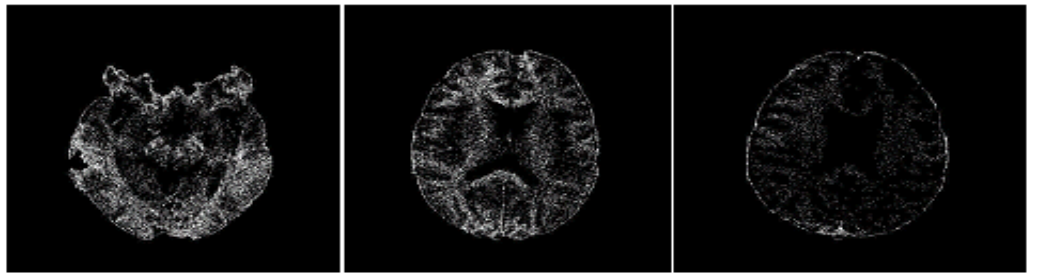

(c)

Figure 7: Segmentation of normal images. (a) WM (b) GM (c) CSF 
The segmentation is performed on these pre-processed images, following the pre-processing of normal images. The normal tissues of these images are White Matter, Gray Matter and Cerebro-Spinal Fluid. WM and GM segmentation is carried out by utilizing Gradient technique and the segmentation of CSF tissue is made by utilizing OPT technique, respectively. The images of normal tissues subsequent to the segmentation procedure are specified in the fig. 7 given below.

\subsection{Performance Evaluation}

By utilizing the performance measures namely False Positive Rate, False Negative Rate, Sensitivity, Specificity and Accuracy, the performance of the system is estimated. The basic count values such as True Positive (TP), True Negative (TN), False Positive (FP) and False Negative (FN) are used by these measures. Both the categorization of normal and abnormal images and the segmentation efficiency of every normal tissue is examined by our proposed work, which are clarified in detail in the next sections.

\subsubsection{Results of Classification Evaluation}

The BrainWeb images include both the normal and abnormal images in our work. These images are categorized into normal and abnormal individually by the procedure of Neuro-Fuzzy classifier. The efficiency of the classifier is examined by the metrics False Positive Rate, False Negative Rate, Sensitivity, Specificity and Accuracy. The explanation of TP, TN, FP, FN values for the categorization of normal and abnormal images is specified in the table I given below.

Table I: Description of TP, TN, FP, FN values for the classification of normal and abnormal images

\begin{tabular}{|c|c|c|}
\hline Description & Classified as normal image & Classified as abnormal image \\
\hline Actually normal image & TP & FN \\
\hline Actually abnormal image & FP & TN \\
\hline
\end{tabular}

False Positive Rate (FPR)

The percentage of cases where an image was classified to normal images, but in fact it did not.

$$
F P R=\frac{F P}{F P+T N}
$$

False Negative Rate (FNR)

The percentage of cases where an image was classified to abnormal images, but in fact it did.

$$
F N R=\frac{F N}{F N+T P}
$$

\section{Sensitivity}

The proportion of actual positives which are correctly identified is the measure of the sensitivity. It relates to the ability of test to identify positive results. 
M.Y.Bhanumurthy and Koteswararao Anne; An efficient neuro-fuzzy based segmentation of normal tissues in brain MRI (BMRI) using extensive feature set, Journal of Biomedical Engineering and Medical Imaging, Volume 1, No 5, Oct (2014) , pp 1-22

$$
\text { Sensitivity }=\frac{\text { Number of true positives }}{\text { Number of true positives }+ \text { Number of false negatives }} \times 100
$$

\section{Specificity}

The proportion of negatives which are correctly identified is the measure of the specificity. It relates to the ability of test to identify negative results.

$$
\text { Specificit } y=\frac{\text { Number of true negatives }}{\text { Number of true negatives }+ \text { Number of false positives }} \times 100
$$

\section{Accuracy}

We can compute the measure of accuracy from the measures of sensitivity and specificity as specified below.

$$
\text { Accuracy }=\frac{T P+T N}{T P+T N+F P+F N} \times 100
$$

Above eqns. (16-20) are as well appropriate for finding the efficiency of segmentation of the tissues WM, GM and CSF.

The subsequent table II explains the categorization efficiency outcomes for the normal and abnormal images with various metric values.

Table II: Effectiveness of classification results using Neuro-Fuzzy classifier for the normal and abnormal images

\begin{tabular}{|c|c|}
\hline Metrics & Values \\
\hline TP & 8 \\
\hline TN & 14 \\
\hline FP & 0 \\
\hline FN & 1 \\
\hline FPR & 0 \\
\hline FNR & 6.667 \\
\hline Sensitivity & $88.9 \%$ \\
\hline Specificity & $100 \%$ \\
\hline Accuracy & $95.65 \%$ \\
\hline
\end{tabular}

In our proposed work, we can establish the efficiency of categorization for the normal and abnormal images by means of Neuro-Fuzzy classifier from the above table II. False Positive Rate and False Negative Rate values are 0 and 6.667, respectively, which explains that our proposed work has low error rate in categorizing images. Properly categorized percentages of normal images are specified by Sensitivity. Neuro-fuzzy classifier offers very high (88.9\%) values for the metric sensitivity, in which only one of the normal image is categorized as abnormal. In addition, Specificity is another metric that specifies the percentage of abnormal images properly categorized. The classifier provides $100 \%$ specificity rate by categorizing the entire actual abnormal images into abnormal images in our work. The highest value in sensitivity and specificity and also the lowest value in the error rates False Positive Rate and False Negative Rate open a mode to raise the categorization correctness outcome with the value $95.65 \%$. Therefore we can show that in proposed work categorization of BMRI BrainWeb images offers high classification accuracy. 


\subsubsection{Results of Segmentation Evaluation}

Every normal image is segmented for the WM, GM and CSF tissues after the categorization. The efficiency of this segmentation of all the tissues are examined by the metrics False Positive Rate, False Negative Rate, Sensitivity, Specificity and Accuracy as specified in the eqns. (16-20). The explanation of TP, TN, FP, FN values for the segmentation of normal image tissues is specified in the following table III.

Table III: Description of TP, TN, FP and FN values for the segmentation of normal images

\begin{tabular}{|c|c|c|}
\hline Description & Segmented as WM tissue & Segmented as not a WM tissue \\
\hline Actually WM tissue & TP & FN \\
\hline Actually not a WM tissue & FP & TN \\
\hline
\end{tabular}

Each image is taking the test either has or does not have the tissue (WM, GM, CSF). The test outcome can be positive (predicting that the image has the particular tissue) or negative (predicting that the image does not have the particular tissue).

Three types of tissues are offered such as WM, GM and CSF in normal images. At first, these three tissues are segmented from the normal images and the segmentation efficiency values are tabularized in the following table IV with different estimation metrics of different images.

Table IV: WM segmentation results for various images

\begin{tabular}{|c|l|c|c|c|c|c|c|c|c|}
\hline Images & TP & TN & FP & FN & FPR & FNR & $\begin{array}{c}\text { Specificity } \\
\text { (in \%) }\end{array}$ & $\begin{array}{c}\text { Sensitivity } \\
\text { (in \%) }\end{array}$ & $\begin{array}{c}\text { Accuracy } \\
\text { (in \%) }\end{array}$ \\
\hline Img 1 & 693 & 253393 & 8058 & 5166 & 0.0308870 & 0.883860 & 96.9179 & 11.8279 & 95.0529348 \\
\hline Img 2 & 1457 & 239434 & 21253 & 3519 & 0.0810368 & 0.704334 & 91.8473 & 29.2805 & 90.6754046 \\
\hline Img 3 & 693 & 256043 & 5408 & 7871 & 0.0209167 & 0.919750 & 97.9315 & 8.09201 & 95.0821251 \\
\hline Img 4 & 1215 & 252815 & 8114 & 6701 & 0.0314603 & 0.850268 & 96.8903 & 15.3486 & 94.4893898 \\
\hline Img 5 & 6512 & 227682 & 27950 & 10063 & 0.1104886 & 0.622534 & 89.0663 & 39.2880 & 86.0352599 \\
\hline
\end{tabular}

Segmentation of White matter gives very good accuracy outcomes. The subsequent fig. 8 explains the corresponding graph for the values in table IV.

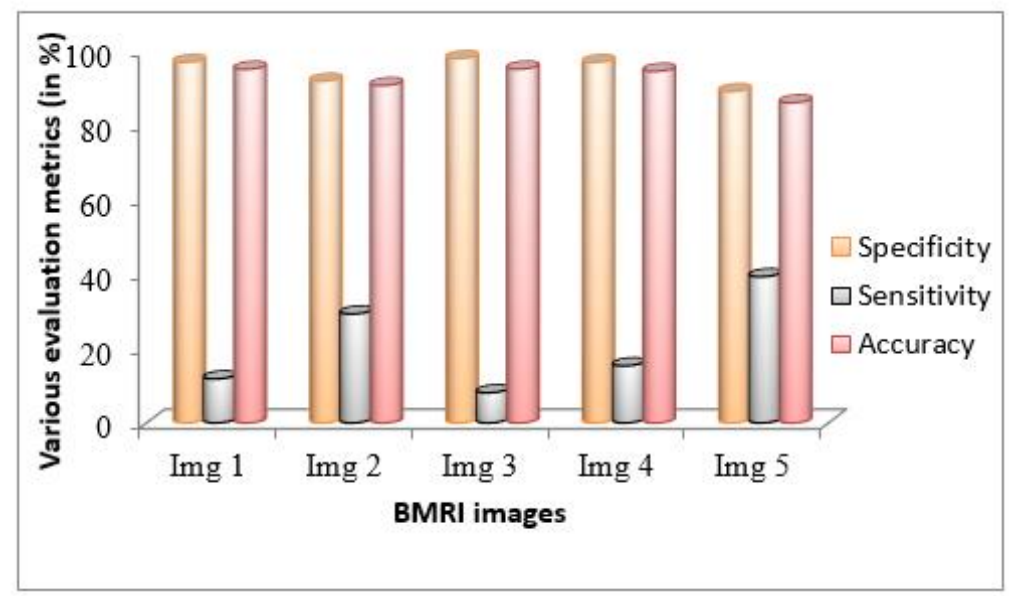

Figure 8: Sensitivity, Specificity and Accuracy results of WM segmentation 
M.Y.Bhanumurthy and Koteswararao Anne; An efficient neuro-fuzzy based segmentation of normal tissues in brain MRI (BMRI) using extensive feature set, Journal of Biomedical Engineering and Medical Imaging, Volume 1, No 5, Oct (2014) , pp 1-22

We can attain the WM segmentation efficiency from the above table IV and its corresponding graph in fig. 8. The FPR and FNR values are very low for our proposed work. These low values offer a way to raise the segmentation accuracy. The specificity for the image 3 is very high (97.93\%) as compared with the other four images. Image 1, 2, 4 and 5 contain 96.91\%, 91.84\%, 96.89\% and $89.06 \%$ of specificity metrics, respectively. These values are also high for our proposed NFBIS, which guides to make high accuracy of segmentation of WM tissue. But the sensitivity values for these five images are low values of $11.82 \%, 29.28 \%, 8.09 \%, 15.34 \%$ and $39.28 \%$ for images $1,2,3,4$ and 5, respectively. Though these values are low, it does not change the segmentation accuracy of WM tissue. Therefore, we can obtain very good accuracy values of $95.05 \%, 90.675 \%, 95.08 \%, 94.48 \%$ and $86.035 \%$ for the images $1,2,3,4$ and 5, respectively. Generally, our proposed work gives $92.264 \%$ of accuracy for the WM tissue segmentation.

The table V shows the GM segmentation outcomes with different images.

Table V: GM segmentation results for various images

\begin{tabular}{|c|c|c|c|c|c|c|c|c|c|}
\hline Images & TP & TN & FP & FN & FPR & FNR & $\begin{array}{c}\text { Specificity } \\
\text { (in \%) }\end{array}$ & $\begin{array}{c}\text { Sensitivity } \\
\text { (in \%) }\end{array}$ & $\begin{array}{c}\text { Accuracy } \\
\text { (in \%) }\end{array}$ \\
\hline Img 1 & 29279 & 211105 & 21760 & 24172 & 0.0903 & 0.451690 & 90.655 & 54.777 & 83.9575 \\
\hline Img 2 & 13731 & 234678 & 13735 & 34343 & 0.0514 & 0.714504 & 94.470 & 28.562 & 83.7841 \\
\hline Img 3 & 55115 & 188526 & 18503 & 9537 & 0.0844 & 0.147501 & 91.062 & 85.248 & 89.6790 \\
\hline Img 4 & 29685 & 221767 & 10692 & 36912 & 0.0470 & 0.555322 & 95.400 & 44.574 & 84.0819 \\
\hline Img 5 & 32333 & 217357 & 12454 & 30587 & 0.0565 & 0.489560 & 94.580 & 51.387 & 85.2967 \\
\hline
\end{tabular}

Corresponding graph of table $V$ is designed in Fig. 9 with different BMRI images for the GM segmentation. The evaluation outcome illustrates whether our proposed work is good or not.

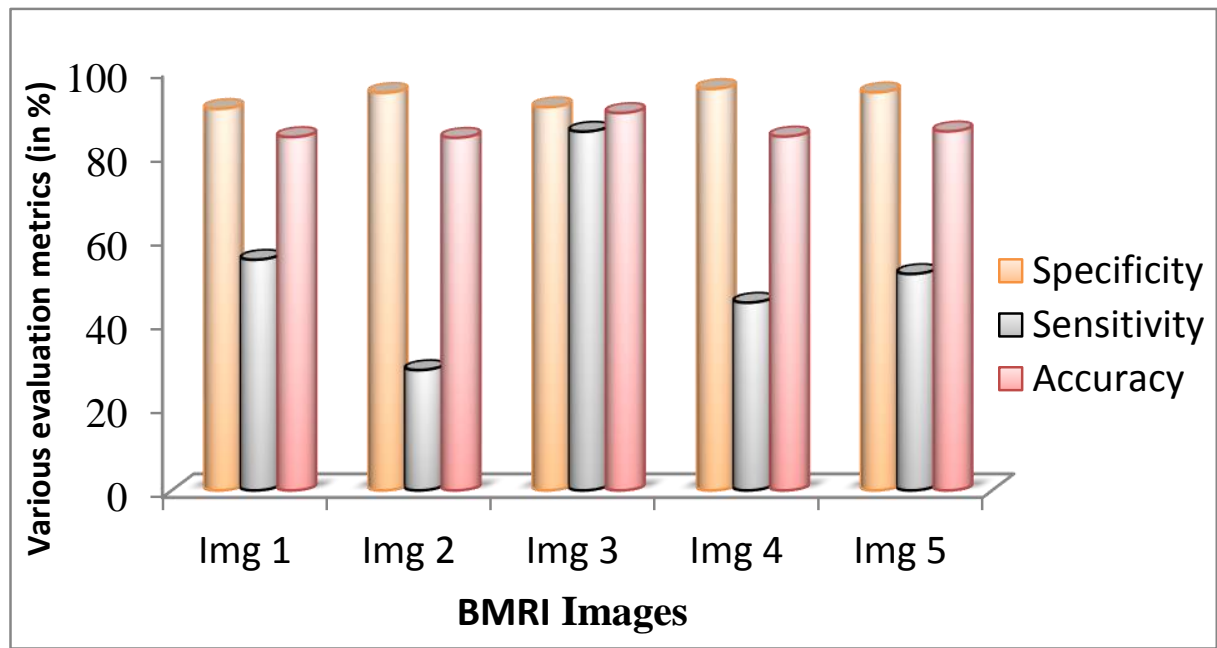

Figure 9: Sensitivity, Specificity and Accuracy results of GM segmentation

We can attain the GM segmentation efficiency from the table $V$ and Fig. 9. The FPR and FNR values are very low for the GM segmentation of our proposed work. The segmentation accuracy can be improved with the help of these low values of FPR and FNR. The specificity for the Images 1, 2, 3, 4 and 5 have $90.65 \%, 94.47 \%, 91.06 \%, 95.4 \%$ and $94.58 \%$ of specificity metric, respectively. These values are high for our proposed NFBIS, which guides to create high accuracy of segmentation of GM tissue. However the 
sensitivity values for these five images are medium values (not very low) of $54.77 \%, 28.56 \%, 85.24 \%$, $44.57 \%$ and $51.38 \%$ for images 1, 2, 3, 4 and 5, respectively. Although these values are not high, it also does not change the segmentation accuracy of GM tissue. Hence, we can achieve good accuracy values of $83.95 \%, 83.78 \%, 89.67 \%, 84.08 \%$ and $85.29 \%$ for the images $1,2,3,4$ and 5, respectively. Overall, our proposed work grants $85.359 \%$ of accuracy for the GM tissue segmentation. Anyway, it is good outcome for our proposed work.

The table VI illustrates the CSF segmentation outcomes with different images.

Table VI: CSF segmentation results for various images

\begin{tabular}{|c|c|c|c|c|c|c|c|c|c|}
\hline Images & TP & TN & FP & FN & FPR & FNR & Specificity (in \%) & Sensitivity (in \%) & Accuracy (in \%) \\
\hline Img 1 & 787 & 256986 & 4371 & 16587 & 0.0167280 & 0.951058 & 98.3275 & 4.5297571 & 92.48092 \\
\hline Img 2 & 2512 & 254993 & 4639 & 31817 & 0.0191407 & 0.928281 & 98.2132 & 7.3174284 & 87.59835 \\
\hline Img 3 & 192 & 260102 & 1850 & 10867 & 0.0070851 & 0.982321 & 99.2937 & 1.7361425 & 95.34194 \\
\hline Img 4 & 541 & 257445 & 4158 & 10166 & 0.0155631 & 0.944853 & 98.4105 & 5.0527692 & 94.73981 \\
\hline Img 5 & 29 & 260976 & 1139 & 1786 & 0.0043074 & 0.985582 & 99.5654 & 1.5977961 & 98.89175 \\
\hline
\end{tabular}

Corresponding graph of table $\mathrm{VI}$ is designed in Fig. 1 with different BMRI images for the CSF segmentation. The evaluation outcomes illustrates whether our proposed work is good or not.

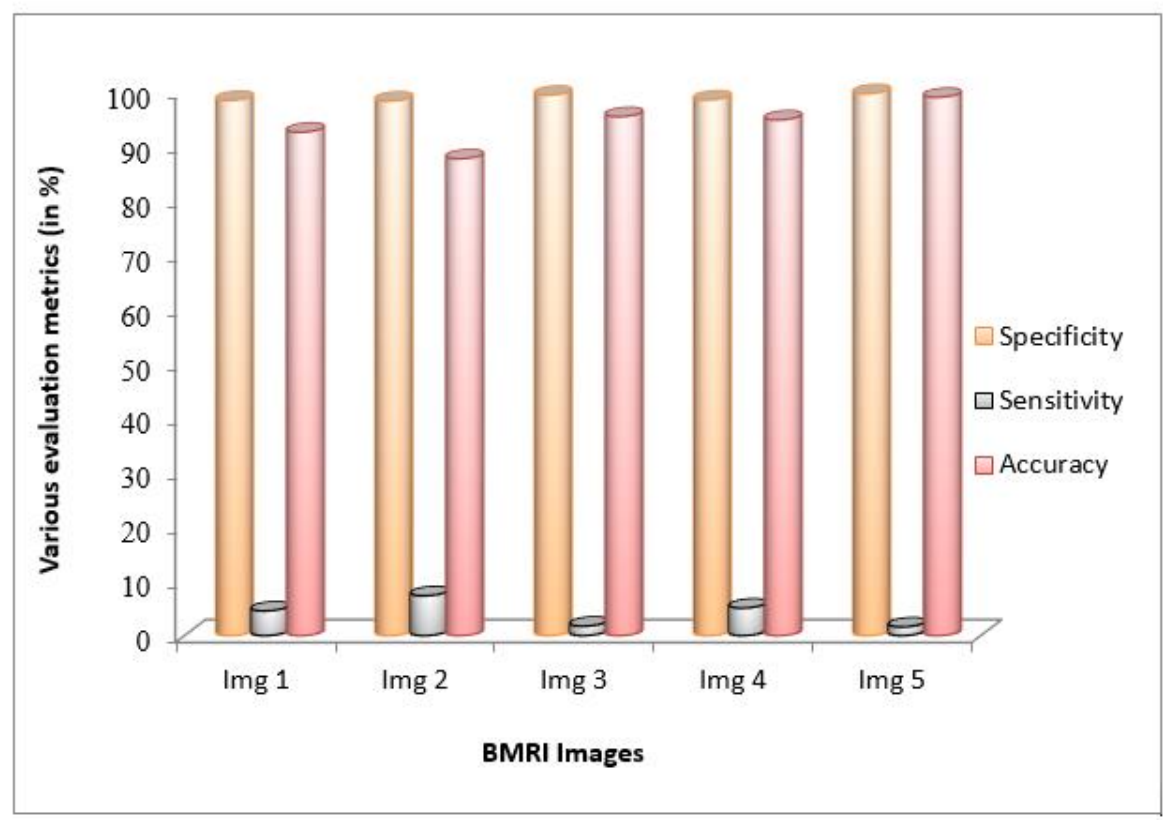

Figure 10: Sensitivity, Specificity and Accuracy results of CSF segmentation

The segmentation of CSF outcomes is attained with diverse evaluation metric values. From the outcomes, it is clearly recognized that our proposed work efficiently segments the CSF tissues by presenting $93.81 \%$ of accuracy on average. This development in the accuracy of CSF segmentation is achieved by the lower error rate values of FPR and FNR. Also, the specificity values of each image are very high by assisting 98.32\%, 98.21\%, 99.29\%, 98.41\% and $99.56 \%$ for image 1, 2, 3, 4, and 5, respectively. However, the sensitivity values are very low for our work. Even these values are low, by no 
M.Y.Bhanumurthy and Koteswararao Anne; An efficient neuro-fuzzy based segmentation of normal tissues in brain MRI (BMRI) using extensive feature set, Journal of Biomedical Engineering and Medical Imaging, Volume 1, No 5, Oct (2014) , pp 1-22

means it lessen the outcomes of segmentation accuracy values. Our proposed work offers low value for the FPR and FNR additionally with this. Moreover it makes the segmentation accuracy of CSF tissue in our proposed work to be improved.

\subsubsection{Comparative Analysis for our proposed work with the existing works}

For the categorization of normal and abnormal brain images our proposed work makes use of NeuroFuzzy classifier. We can establish that our proposed work helps to attain very good accuracy for the categorization of images utilizing Neuro-Fuzzy classifier from the above sections 4.2 .1 and 4.2.2. And also we can establish this categorization accuracy outcome by comparing other classifiers. We have utilized Artificial Neural Network and Fuzzy C-Means for our comparison in our work. The comparison outcomes are presented in the following table VII.

Table VII: Comparison results for the image classification with other classifiers

\begin{tabular}{|c|c|c|c|}
\hline Metrics & Fuzzy C-Means & Artificial Neural Network & $\begin{array}{c}\text { Neuro-Fuzzy in our } \\
\text { proposed work }\end{array}$ \\
\hline TP & 0 & 1 & 8 \\
\hline TN & 14 & 9 & 14 \\
\hline FP & 0 & 5 & 1 \\
\hline FN & 9 & 8 & 0 \\
\hline FPR & 0 & 0.3571 & 6.667 \\
\hline FNR & 0.3913 & 0.4706 & 88.9 \\
\hline Sensitivity (in \%) & 0 & 11.11 & 100 \\
\hline Specificity (in \%) & 100 & 64.29 & 95.65 \\
\hline Accuracy (in \%) & 60.87 & 43.48 & \\
\hline
\end{tabular}

Below specified fig. 11 explains the comparison outcomes of the classifiers for the BMRI image categorization with different metrics. The improved accuracy outcomes of categorization of BMRI images into normal and abnormal images are presented by our proposed work. In comparison with the classifier Neuro-Fuzzy, both the Fuzzy C-Means and Artificial Neural Networks gives very less accuracy values for the categorization of images. The sensitivity for the Fuzzy C-Means and Artificial Neural Networks are $0 \%$ and $11.11 \%$, which is low in compared with our classifier, Neuro-Fuzzy $88.9 \%$. The specificity is $100 \%$ for our classifier and for the Fuzzy C-means classifier. However the accuracy is $95.65 \%$ for our Neuro-fuzzy classifier and the fuzzy C-means and ANN contain only low categorization accuracy results of $60.87 \%$ and $43.48 \%$, respectively. From these outcomes, it is known that by means of NeuroFuzzy classifier in our work provides very good for the categorization purpose as it gives improved accuracy outcomes. Therefore, our work shows that it is worth for the categorization and segmentation of BMRI images. 


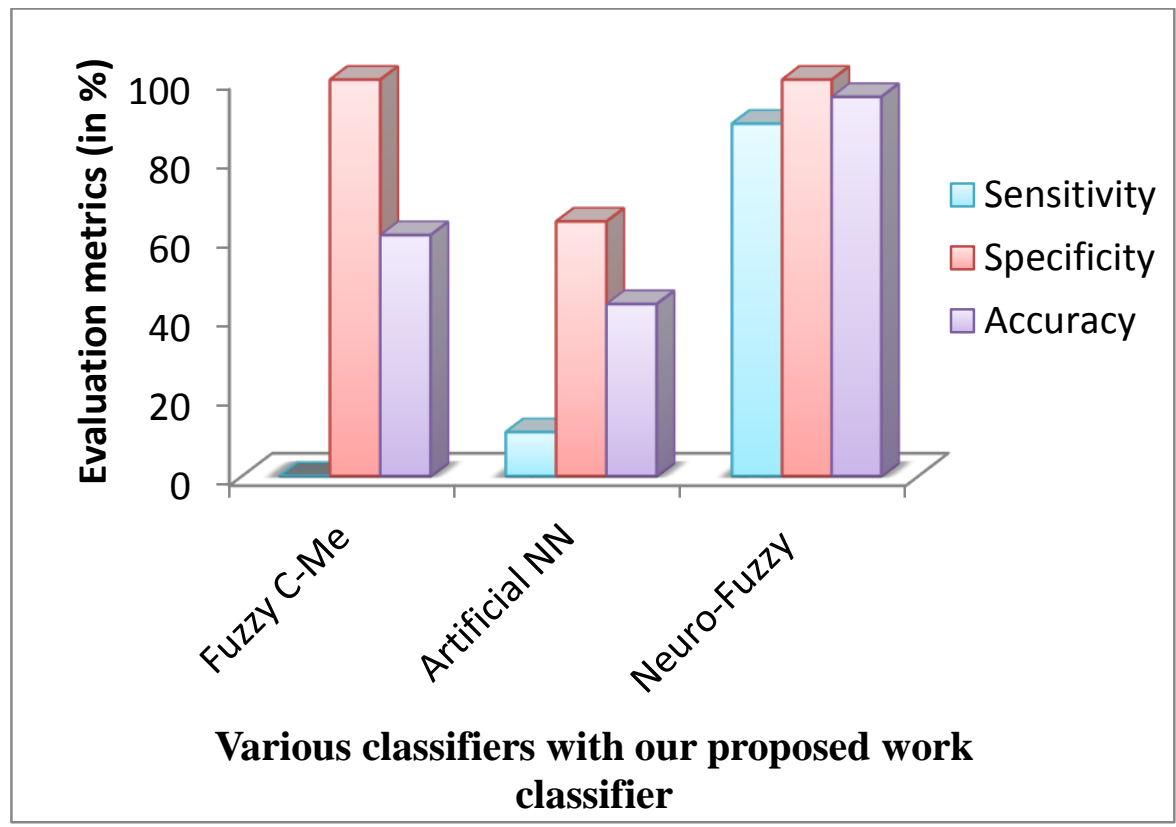

Figure 11: Comparison graph for the image classification with other classifiers

\section{CONCLUSION}

A Neuro Fuzzy based BMRI image segmentation technique with three phases - Feature Extraction, Classification and Segmentation was proposed in this paper. The features from the BMRI images were extracted and then specified to the Neuro-Fuzzy classifier. The classification of normal and abnormal images was made by this Neuro-Fuzzy classifier and these classified images were segmented efficiently by our proposed method. The testing was performed with the BrainWeb images dataset. The performance measures False Positive Rate, False Negative Rate, sensitivity, specificity and accuracy were evaluated for our proposed method. The testing was prepared for establishing the accuracy of both the classification of images into normal and abnormal and segmentation of every normal tissue. The efficiency of the classification of images is very high by presenting very good accuracy outcomes and also the segmentation of each tissue offers very accurate outcomes. From the outcomes, we have showed that the Neuro-Fuzzy classifier utilized in our proposed work outperforms the other classifiers Fuzzy C-Means and ANN by facilitated very good accuracy of $95.65 \%$ in categorizing the images into normal and abnormal.

\section{REFERENCES}

[1]. P. Hagmann, J.P. Thiran, L. Jonasson, "DTI mapping of human brain connectivity: statistical fibre tracking and virtual dissection", Neurolmage, 2003.

[2]. M.C. Davidson, K. M. Thomas. and B. J. Casey, "Imaging the developing brain with fMRI", Mental Retardation and developmental disabilities research reviews, 2003.

[3]. V. A. Grau, U. J. Mewes, M. Alcaniz, "Improved watershed transform for medical image segmentation using prior information", IEEE Trans. on Medical Imaging, 2004, 23(4): 447-458 
M.Y.Bhanumurthy and Koteswararao Anne; An efficient neuro-fuzzy based segmentation of normal tissues in brain MRI (BMRI) using extensive feature set, Journal of Biomedical Engineering and Medical Imaging, Volume 1, No 5, Oct (2014) , pp 1-22

[4]. H Lv., K. H. Yuan, S. L. Bao, "An eSnake model for medical imaging segmentation", Progress in Natural Science, 2005.

[5]. D. L. Pham and J. L. Prince, "Adaptive fuzzy segmentation of magnetic resonance images," IEEE Trans. Med. Imag., 1999.

[6]. A. F. Goldszal, C. Davatzikos, D. L. Pham, M. X. H. Yan, et al, "An image processing system for qualitative and quantitative volumetric analysis of brain images," J. Comput. Assist. Tomogra., 1998.

[7]. $\quad$ Arnold J.B., Liow, J.-S., Schaper, K.A., et al., "Qualitative and quantitative evaluation of six algorithms for correcting intensity nonuniformity effects". Neurolmage, 2001.

[8]. R. Moller., R. Zeipelt. "Automatic segmentation of 3D-MRI data using a genetic algorithm, Medical Imaging and Augmented Reality", 2001. Proceedings. International Workshop on, 10-12 June 2001:278 $-281$.

[9]. W. M.Wells, III,W. E. L. Grimson, R. Kikinis. "Adaptive segmentation of MRI data”, IEEE Trans. Medical Imaging , 1996.

[10]. J. C. Bezdek, L.O. Hall, L. P. Clarke, "Review of MR image segmentation techniques using pattern recognition," Med. Phys., vol. 20, No. 4, pp. 1033-1048, 1993.

[11]. Suetens, E. Bellon, D. Vandermeulen, M. Smet, G. Marchal, J. Nuyts, L. Mortelman, "Image segmentation: methods and applications in diagnostic radiology and nuclear medicine," European Journal of Radiology, vol. 17, pp. 14-21, 1993.

[12]. A. Goshtasby, D. A. Turner, "Segmentation of Cardiac Cine MR Images for extraction of right and left ventricular chambers," IEEE sTrans. Med. Imag., vol. 14, No. 1, pp. 56-64, 1995.

[13]. D. Brzakovic, X. M. Luo, P. Brzakovic, "An approach to automated detection of tumors in mammograms," IEEE Trans. Med. Imag., vol. 9, No. 3, pp. 233-241, 1990.

[14]. J. F. Brenner, J. M. Lester, W.D. Selles, "Scene segmentation in automated histopathology: techniques evolved from cytology automation," Pattern Recognition, vol. 13, pp. 65-77, 1981.

[15]. K. Lim, A. Pfefferbaum, "Segmentation of MR brain images into cerebrospinal fl-uid spaces, white and gray matter," J. Comput. Assist. Tomogr., vol. 13, pp. 588-593, 1989.

[16]. Zhang Y, Brady M, Smith S. "Segmentation of brain MR images through a hidden Markov random field model and expectation-maximization algorithm,". IEEE Trans Med. Imag., pp. 45-57, 2001.

[17]. L. Lemieux, G. Hagemann, K. Krakow, and F. G. Woermann, "Fast, accurate, and reproducible automatic segmentation of the brain in T1-weighted volume MRI data, "Magn. Reson. Med., vol. 42, pp. 127-135, 1999.

[18]. R. Pohle and K. D. Toennies, "Segmentation of medical images using adaptive region growing," Proc. SPIE-Med. Imag., vol. 4322, pp. 1337-1346, 2001.

[19]. S. Shen, W Sandham, M. Grant and A. Ster, "MRI Fuzzy Segmentation of Brain Tissue Using Neighborhood Attraction with Neural Network Optimization", IEEE Trans. On Information Technologyis Biomedicine, vol. 9, No. 3, 2005. 
[20]. Arnaldo Mayer and Hayit Greenspan, "An Adaptive Mean-Shift Framework for MRI Brain Segmentation", IEEE Transactions On Medical Imaging, Vol. 28, No. 8, August 2009.

[21]. Mert R. Sabuncu, B.T. Thomas Yeo, Koen Van Leemput, Bruce Fischl and Polina Golland, "A Generative Model for Image Segmentation Based on Label Fusion", leee Transactions On Medical Imaging, 2009.

[22]. Feng Shi, Yong Fan, Songyuan Tang, John H. Gilmore, Weili Lin, Dinggang Shen, "Neonatal brain image segmentation in longitudinal MRI studies", Elsevier Inc., 2009.

[23]. Juin-Der Lee, Hong-Ren Su, Philip E. Cheng*, Michelle Liou, John A. D. Aston, Arthur C. Tsai, and ChengYu Chen, "MR Image Segmentation Using a Power Transformation Approach", IEEE Transactions On Medical Imaging, Vol. 28, No. 6, June 2009.

[24]. Dalila Cherifi, M.Zinelabidine Doghmane, Amine Nait-Ali, Zakia Aici,Salim Bouzelha, "Abnormal tissus extraction in MRI Brain medical images", IEEE, 2011.

[25]. Nagesh Vadaparthi, Srinivas Yarramalle, Suresh Varma Penumatsa, "Unsupervised Medical Image Segmentation On Brain MRI Images Using Skew Gaussian Distribution", IEEE-International Conference on Recent Trends in Information Technology, ICRTIT 2011.

[26]. Soumya Maitra, "Morphological Edge Detection Using Bit-Plane Decomposition in Gray Scale Images", In Proceedings of INDIACom, 2011. 